\title{
On the Alternatives of Lyapunov's Direct Method in Adaptive Control Design
}

\author{
Hamza Khan, József K Tar* and Imre J Rudas \\ Óbuda University, Hungary
}

Submission: October 14, 2018; Published: October 26, 2018

*Corresponding author: József K Tar, Óbuda University, Hungary; Tel: +36-30-330-3022; Email: tar.jozsef@nik.uni-obuda.hu

\begin{abstract}
The prevailing methodology in designing adaptive controllers for strongly nonlinear systems is based on Lyapunov's PhD Thesis he defended in 1892 to study the stability of motion of systems for the solution of the equations of motion of which no closed form analytical solutions exist. The adaptive robot controllers developed in the nineties of the 20thcentury guarantee global (often asymptotic) stability of the controlled system by using his ingenious Direct Method that introduces a Lyapunov function for the behavior of which relatively simple numerical limitations have to be proved. Though for various problem classes typical Lyapunov function candidates are available, the application of this method requires far more knowledge than the implementation of some algorithm. Besides requiring creative designer's abilities, it often requires too much because it works with satisfactory conditionsinstead of necessary and satisfactoryones. To evade these difficulties, based on the firm mathematical background of constructing convergent iterative sequences by contractive maps in Banach spaces, an alternative of Lyapunov's technique was so introduced for digital controllers in 2008 that during one control cycle only one step of the required iteration was done. Besides its simplicity the main advantage of this approach was the possible evasion of complete state estimation that normally is required in the Lyapunov functionbased design. Though the convergence of the control sequence can be guaranteed only within a bounded basin, this approach seems to have considerable advantages. In the paper the current state of the art of this approach is briefly summarized.
\end{abstract}

Keywords: Adaptive control; Lyapunov function; Banach space; Fixed point lteration

Abbreviations: AC: Adaptive Control; AFC: Acceleration Feedback Controller; AID: Adaptive Inverse Dynamics Controller; CTC: Computed Torque Control; FPI: Fixed Point Iteration; MRAC: Model Reference Adaptive Control; OC: Optimal Control; PID:Proportional, Integrated, Derivative;

RARC: Resolved Acceleration Rate Control; RHC: Receding Horizon Controller;SLAC: Slotine-Li Adaptive Controller;

\section{Introduction}

There is a wide class of model-based control approaches in which the available approximate dynamic model of the system to be controlled is "directly used" without "being inserted" into the mathematical framework of "Optimal Control" (OC). A classical example is the "Computed Torque Control" (CTC) for robots [1]. However, in the practice we have to cope with the problem of the imprecision (very often incompleteness) of the available system models (in robotics e.g. [1,2], modeling friction phenomena e.g. [37], in life sciences as modeling the glucose-insulin dynamics e.g. [811] or in anesthesia control e.g. [12-14]). Modeling such engines as aircraft turbojet motors is a quite complicated task that may need multiple model approach [15-18]. Further practical problem is the existence and the consequences of unknown and unpredictable "external disturbances". A possible way of coping with these practical difficulties is designing "Adaptive Controllers" (AC) that somehow are able to observe and correct at least the effects of the modeling imprecisions by "learning". Depending on the above available information on the model various adaptive methods can be elaborated. If we have precise information on the kinematics of a robot and only approximate information is available on the mass distribution of a robot arm made of rigid links the exact model parameters can be learned as in the case of the "Adaptive Inverse Dynamics" (AID) and the "Slotine-Li Adaptive Controller" (SLAC) for robots that are the direct adaptive extensions of the CTC control. An alternative approach is the adaptive modification of the feedback gains or terms [19]. The "Model Reference Adaptive Control" (MRAC) has double "intent": a) it has to provide precise trajectory tracking, and b) for an outer, kinematics-based control loop they have to provide an illusion that instead of the actually controlled system, a so called "reference system" is under control (e.g. [20-22]).

The traditional approaches in controller design for strongly nonlinear systems are based on the PhD thesis by Lyapunov [23] that later was translated to Western languages (e.g. [24]). (In this context "strong nonlinearity" means that the use of a "linearized system model" in the vicinity of some "working point" 


\section{Robotics \& Automation Engineering Journal}

is not satisfactory for practical use.) In Lyapunov's " 2 nd" or "Direct Method" a Lyapunov function has to be constructed for the given particular problem (typical "candidates" are available for typical "problem classes"), and non-positiveness of the time-derivative of this function has to be proved. Besides the fact that the creation of the Lyapunov function is not a simple application of some algorithm -it is rather some creative art-, this method has various drawbacks as a) it works with "satisfactory conditions" instead of "necessary and satisfactory conditions" (i.e. often it requires too much as guaranteeing really not necessary conditions), b) its main emphasis is on global (asymptotic) stability of the motion of the controlled system without paying too much attention to the "initial" or "transient" phase of the controlled motion (for instance in life sciences a "transient" fluctuation can be lethal).

To cope with these difficulties alternatives of the Lyapunov function-based adaptive design were suggested in [25] in which the primary design intent is keeping at bay the initial "transients" by turning the task of finding the necessary control signal to iteratively so solving a fixed point problem ["Fixed Point Iteration" (FPI)] that in each digital control step only one step of the appropriate iteration can be realized. The mathematical antecedents of this approach were established in the $17^{\text {th }}$ century (e.g. [26-28]), and its foundations in 1922 were extended to quite complicated spaces by Stefan Banach [29,30]. In [25] the novelty was the application of this approach to control problems. In contrast to the "traditional" "Resolved Acceleration Rate Control" (RARC) in which in the control of a $2^{\text {nd }}$ order physical system only lower order derivatives or tracking error integrals are fed back (e.g. [19,31-33]) in this approach the measured "acceleration" signals are also used as in the "Acceleration Feedback Controllers" (AFC) (e.g. [34-38]).

In general, the most important "weak point" of the FPI-based approach is that it cannot guarantee global stability. The generated iterative control sequences converge to the solution of the control task only within a bounded basin that in principle can be left. To avoid this problem heuristic tuning rules were introduced for one of the little numbers of the adaptive parameters in [39-41]. In [42] essentially the same method was introduced in the design of a novel type of MRAC controllers the applicability of which was investigated by simulations for the control of various systems (e.g. [43-46]). Observing the fact that in the classical, Lyapunov function-based solutions as the AID and SLAC controllers the parameter tuning rule obtained from the Lyapunov function has a simple geometric interpretation that is independent of the Lyapunov function itself, the FPI-based solution was combined with the tuning rule of the original solutions used for learning the "exact dynamic parameters" of the controlled system. Alleviated from the burden of necessarily constructing some easily treatable quadratic Lyapunov function, the feedback provided by the FPIbased solution was directly used for parameter tuning. This solution resulted in precise trajectory tracking even in the initial phase of the learning process in which the available approximate model parameters still were very imprecise $[47,48]$. In the present paper certain novel results are summarized on the further development of the FPI-based approach.

\section{Discussion and Results}

\section{The structure of the FPI-based adaptive control}

The block scheme of the FPI-based adaptive controller is given in Figure 1 for a $2^{\text {nd }}$ order dynamical system as e.g. a robot [48]. In this case the $2^{\text {nd }}$ time-derivative of the generalized coordinates (joint coordinates). $\ddot{q}$ can be instantaneously set by the control torque or force $Q$ on this basis, in the kinematic block an arbitrary desired joint acceleration $\ddot{q}^{\text {Des }}$ can be designed that can drive the tracking error ${ }_{q}{ }^{N}(t)-q(t)$ to 0 if it is realized. In the practice this joint acceleration cannot be realized due to the imprecisions in the dynamic model the CTC controller uses for the calculation of the necessary forces. Therefore, instead introducing this signal into the Approximate Model to calculate the necessary force its deformed version, $\ddot{q}^{\text {Def }}$ is introduced into it. The necessary deformation iteratively is produced in the form of a sequence that is initiated by it, i.e. by $\ddot{q}^{\text {Def }}$ During one digital control step one step of the iteration can be realized. If there are no special time-delay effects in the system, the contents of the delay boxes in Figure 1 exactly correspond to the cycle time of the controller $\Delta t$ The "chain of operations" resulting in an observed realized response $\ddot{q}(t)$ for the input $\ddot{q}^{D e f}$ mathematically approximately can be considered as a response $\ddot{q}=f\left(\ddot{q}^{\text {Def }}\right)$ since -though it depends on $q$ and $\dot{q}$ - only slowly varies in comparison to " $\ddot{q}^{\text {Def }}$ that quite quickly can be modified. In the Adaptive Deformation Block of Figure 1 a function is used as $\ddot{q}^{D e f}(t+\Delta t)=G\left(\ddot{q}^{D e f}(t), \ddot{q}(t), \ddot{q}^{D e s}(t+\Delta t)\right)$ in which $\ddot{q}(t)=f\left(\ddot{q}^{D e f}(t)\right)$ [49]. Since due to the proportional, integral and derivative error feedback terms ${ }_{\ddot{q}}$ Des varies only slowly, we have an approximation as $\ddot{q}^{\text {Def }}(t+\Delta t)=F\left(\ddot{q}^{\text {Def }}(t)\right)$. Regarding the convergence of this iteration, we have to take it into account that a Banach Space (accidentally denoted by B is a complete, linear, normed metric space. It is a convenient modeling tool that allows the use of simple norm estimations. Its completeness means that each self-convergent or Cauchy sequence has a limit point within the space. A mapping $F: \beta \mapsto \beta$ is contractive if $\exists$ a real number $0 \leq K<1$ so that, $\|F(y)-F(x)\| \leq K\|y-x\| \forall x, y \varepsilon \beta$ It is easy to show that the sequence generated by a contractive map as $\left\{x_{0}, x_{1}=F\left(x_{0}\right), \ldots, x_{n+1}=F\left(x_{n}\right), \ldots\right\}$ is a Cauchy sequence: in the norm estimation given in (1) $\forall L \varepsilon$ in high order powers of $\mathrm{K}$ occur as $n \rightarrow \infty$ therefore $\left\|x_{n}+L-x_{n}\right\| \rightarrow 0$. Due to the completeness of $\beta \exists x_{*} \varepsilon \beta$ so that $x_{n} \rightarrow x_{*}$ It is easy to prove that $F\left(x_{*}\right)=x_{*}$ : for an arbitrary element of the sequence $x_{n}$ according to (2) it holds that $\forall \varepsilon>0\left\|F\left(x_{*}\right)-x_{*}\right\|<\varepsilon$.

$$
\begin{aligned}
& \left\|x_{n+L}-x_{n}\right\|=\left\|F\left(x_{n-1+L}\right)-F\left(x_{n-1}\right)\right\| \leq \\
& K\left\|x_{n-1+L}-x_{n-1}\right\|=K\left\|F\left(x_{n-2+L}\right)-F x_{n-2}\right\| \leq \\
& K^{2}\left\|x_{n-2+L}-x_{n-2}\right\| \text { etc ....................... (1a) }
\end{aligned}
$$




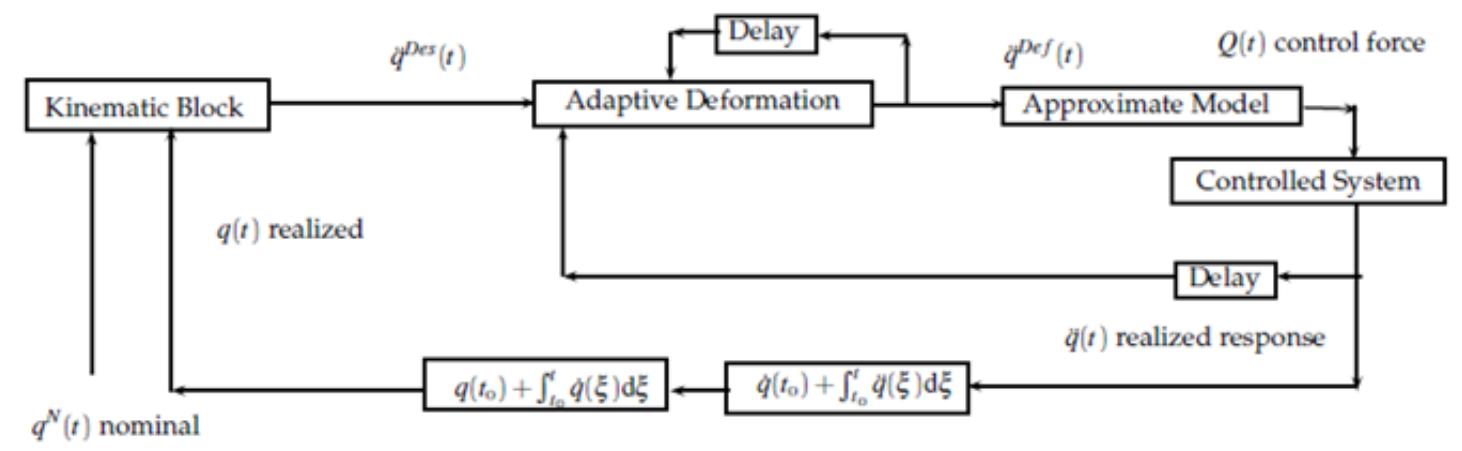

Figure 1: Schematic structure of the "Fixed Point Transformation-based Adaptive Controller" taken from [49].

$$
\begin{aligned}
& \left\|F\left(x_{*}\right)-x_{*}\right\|=\left\|F\left(x_{*}\right)-\left(x_{n}-x_{*}\right)\right\|= \\
& \leq\left\|F\left(x_{*}\right)-F\left(x_{n-1}\right)\right\|+\left\|x_{n}-x_{*}\right\| \leq \\
& \leq K\left\|x_{*}-x_{n-1}\right\|+\left\|x_{n}-x_{*}\right\| \rightarrow 0 \text { as } n \rightarrow \infty
\end{aligned}
$$

Consequently, it is enough to guarantee that the function F(.) is contractive, since in this case the sequence converges to the fixed point of this function if it is so constructed that its fixed point is the solution of the control task.

\section{Construction of the adaptive function}

In the original solution in [25] (3) was suggested for the special case $q \varepsilon I R$ with three adaptive parameters $\mathrm{K}_{c^{\prime}} \mathrm{B}_{c^{\prime}}$ and $\mathrm{A}_{c^{\prime}}$.

$\ddot{q}^{\text {Def }}(t+\Delta t)=\left(K_{c}+\ddot{q}^{\text {Def }}(t)\right)\left[1+B_{c} \tanh \left(A_{c}\left(f\left(\ddot{q}^{\text {Def }}(t)\right)-\ddot{q}^{\text {Des }}(t+\Delta t)\right)\right]-K_{c} \cdots\right.$

Really, when $f\left(\ddot{q}^{\text {Def }}(t)\right)-\ddot{q}^{\text {Def }}(t+\Delta t)=0$ we just have the solution of the control task and it is obtained that $\ddot{q}^{\text {Def }}(t+\Delta t)=\ddot{q}^{\text {Def }}(t)=f\left(\ddot{q}_{*}^{\text {Def }}\right)=\ddot{q}^{\text {Def }}(t+\Delta t)$, that is the solution is a fixed point. To obtain convergence in the vicinity of the fixed consider the 1st order Taylor series approximation as

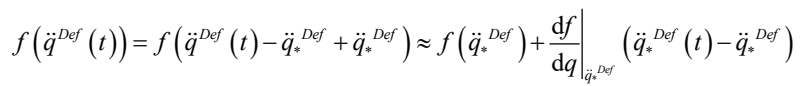

leads to the approximation

$$
\ddot{q}^{\text {Def }}(t+\Delta t)-\ddot{q}_{*}^{\text {Def }} \approx\left[1+\left.\left(K_{c}+\ddot{q}^{\text {Def }}(t)\right) B_{c} A_{c} \frac{\mathrm{d} f}{\mathrm{~d} q}\right|_{\ddot{q}_{*}^{\text {Def }}}\right]\left(\ddot{q}_{*}^{\text {Def }}(t)-\ddot{q}_{*}^{\text {Def }}\right)
$$

On the basis of (5) it is easy to set the adaptive parameters for convergence: by choosing a great parameter $K_{c} \geq\left|\ddot{q}^{\text {Def }}\right|, B_{c}= \pm 1$ and a small Ac it can be achieved that $\left|\ddot{q}^{\text {Def }}(t+\Delta t)-\ddot{q}_{*}^{\text {Def }}\right|<$ therefore the mapping is contractive and the sequence converges to the solution. The speed of convergence depends on setting Ac, and too great value can cause leaving the region of convergence.

For $q \varepsilon I R^{n}$ (multiple variable systems) a different construction was introduced in $[50,51]$ the convergence properties of which were more lucid than that of the multiple variable variant of (3): $\ddot{q}^{D e f}(t+\Delta t) \underline{\underline{\operatorname{def}}}\left[\zeta\left(A_{c}\|h(t)\|+x_{*}\right)-x_{*}\right] e(t)+\ddot{q}^{D e f}(t) \cdots(6)$

in which the expression $h(t) \operatorname{def} f\left(\ddot{q}^{\text {Def }}(t)\right)-\ddot{q}^{\text {Des }}(t+\Delta t)$ can be identified as the "response error in time $t$ ", and with the Frobenius norm $e(t) \underline{\underline{d e f}} \frac{h(t)}{\|h(t)\|}$ corresponds to the unit vector that is directed into the direction of the response error, $\zeta: I R \mapsto I R$ is a differentiable contractive map with the attractive fixed point $\zeta\left(x_{*}\right)=x_{*}$ and $A_{c} \varepsilon I R$ is an adaptive control parameter. By using the same argumentation with the $1^{\text {st }}$ order Taylor series approximation it was shown in [52] that if the real part of each eigenvalue of $\frac{\partial f}{\partial \dot{q}^{D f f}}$ is simultaneously positive or negative, an appropriate $\mathrm{A}_{\mathrm{c}}$ parameter can be selected that guarantees convergence.

$m_{1} \ddot{q}_{1}=m_{1} g-k_{1} \operatorname{sign}\left(q_{1}-L_{01}\right)\left|q_{1}-L_{01}\right|^{\sigma_{1}}+k_{2} \operatorname{sign}\left(q_{2}-q_{1}-L_{02}\right)\left|q_{2}-q_{1}-L_{02}\right|^{\sigma_{2}}-b_{1^{\prime}}$ (7)

in which $Q \varepsilon I R^{2}$ denotes the control force and $q \varepsilon I R^{2}$ is the array of the generalized coordinates of the controlled system.

The parameter $\sigma_{1}$, and $\sigma_{2}>0$ "modulate" the springs' stiffness, the direction of the spring force is calculated by the use of the "signum" function as sign $\left(q_{1}-L_{01}\right)$ while its absolute value is $\left|q_{1}-L_{01}\right|^{\sigma_{1}}$. The approximate and exact model parameter values are given in Table 1.

Table 1: The system's exact and approximate parameter values.

\begin{tabular}{|c|c|c|}
\hline Parameter & Approximate value & Exact value \\
\hline Mass of $\mathrm{m}_{1}[\mathrm{~kg}]$ & 1.5 & 1.0 \\
\hline Mass of $\mathrm{m}_{2}[\mathrm{~kg}]$ & 2.6 & 2.0 \\
\hline Nonlinearity of spring $1 \sigma_{1}[$ dimless $]$ & 1.5 & 2.0 \\
\hline Nonlinearity of spring $2 \sigma_{2}[$ dimless $]$ & 1.0 & 2.5 \\
\hline
\end{tabular}


Robotics \& Automation Engineering Journal

\begin{tabular}{|c|c|c|}
\hline Spring's stiffness of $k_{1}\left[\frac{N}{m}\right]$ & 120.0 & 100.0 \\
\hline Spring's stiffness of $k_{2}\left[\frac{N}{m}\right]$ & 165.0 & 150.0 \\
\hline Length of $L_{01}[\mathrm{~m}]$ in zero force case & 1.9 & 3.0 \\
\hline Length of $L_{02}[\mathrm{~m}]$ in zero force case & 3.1 & 0.1 \\
\hline Viscous friction coefficient b1 ${ }^{b_{1}\left[\frac{N-s}{m}\right]}$ & 0.2 & 2.0 \\
\hline Viscous friction coefficient b2 ${ }^{2}\left[\frac{N-s}{m}\right]$ & 2.5 & 10 -Mar \\
\hline Gravitational acceleration g $g\left[\frac{m}{s^{2}}\right]$ & 10.0 & Not Applicable \\
\hline Time resolution and cycle time of simulation & $\Delta t[s]$ & \\
\hline
\end{tabular}

In the Kinematic Block for the integrated error $e_{i \text { int }}(t) d e f \int_{f_{n}}\left[q^{N}(\xi)\right] d \xi$ the prescribed "tracking strategy" was $\left(\frac{d}{d t}+\right)^{3} e_{e_{m}(t)=0}$ that lead to a PID-type feedback $\ddot{q}^{D e s}(t)=\ddot{q}^{N}(t) \wedge^{3} e_{\text {int }}(t)+3 \wedge^{2} e(t)+3 \wedge \dot{e}(t)$ that choice guarantees the convergence $q(t) \rightarrow q^{N}(t)$ as $t \rightarrow \infty$ in the simulations $\wedge=6 s^{-1}$ was chosen with $\zeta(x)=\operatorname{atanh}(\tanh (x+D) / 2), D=0.3$ in (6). The choice $A_{c}=-5 \times 10^{-1}$ resulted in good convergence. The Figure2-6 illustrate the effects of using the adaptive deformation. It is evident that the tracking precision was considerably improved without any chattering effect that are typical in the also simple Sliding Mode / Variable Structure Controllers (e.g. [53,54]. Figure 5 reveals that quite different control forces were applied in the non-adaptive and in the adaptive cases.
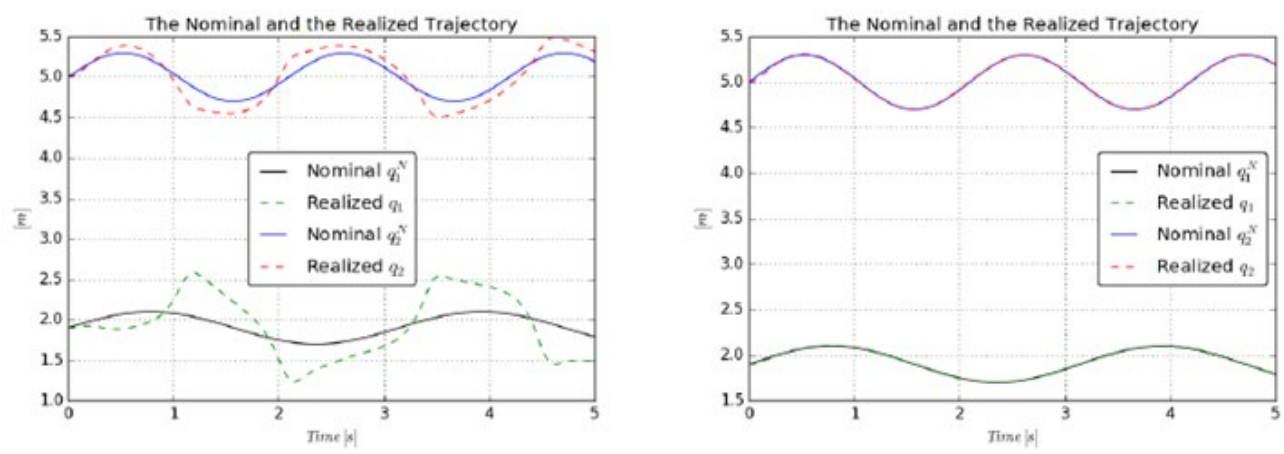

Figure 2: Trajectory tracking without adaptivity (LHS) and with adaptivity (RHS).
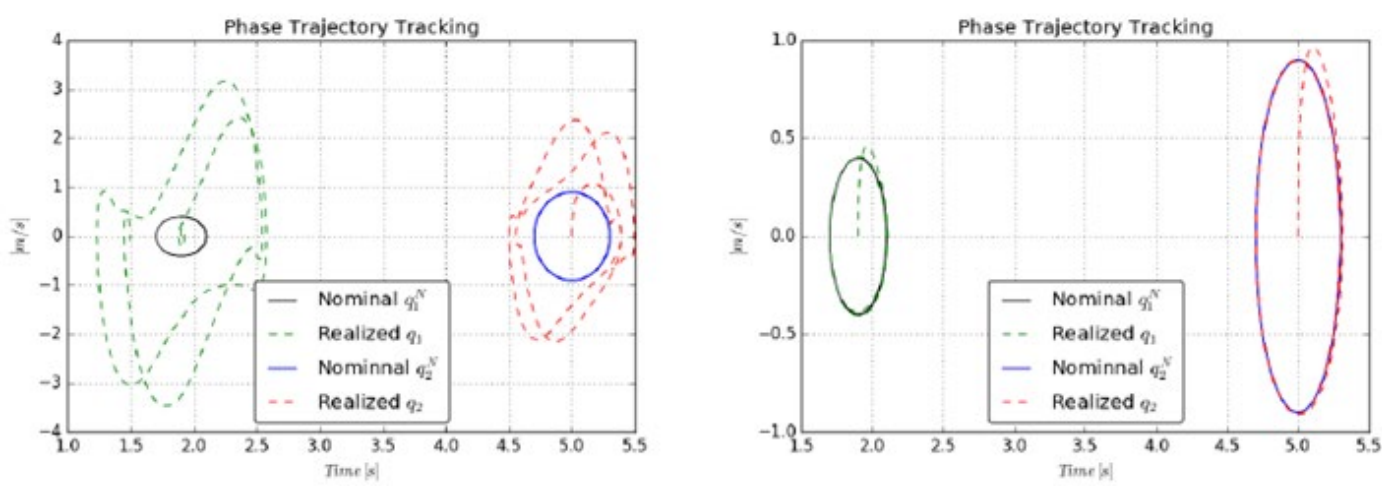

Figure 3: Phase Trajectory tracking without adaptivity (LHS) and with adaptivity (RHS). 


\section{Robotics \& Automation Engineering Journal}
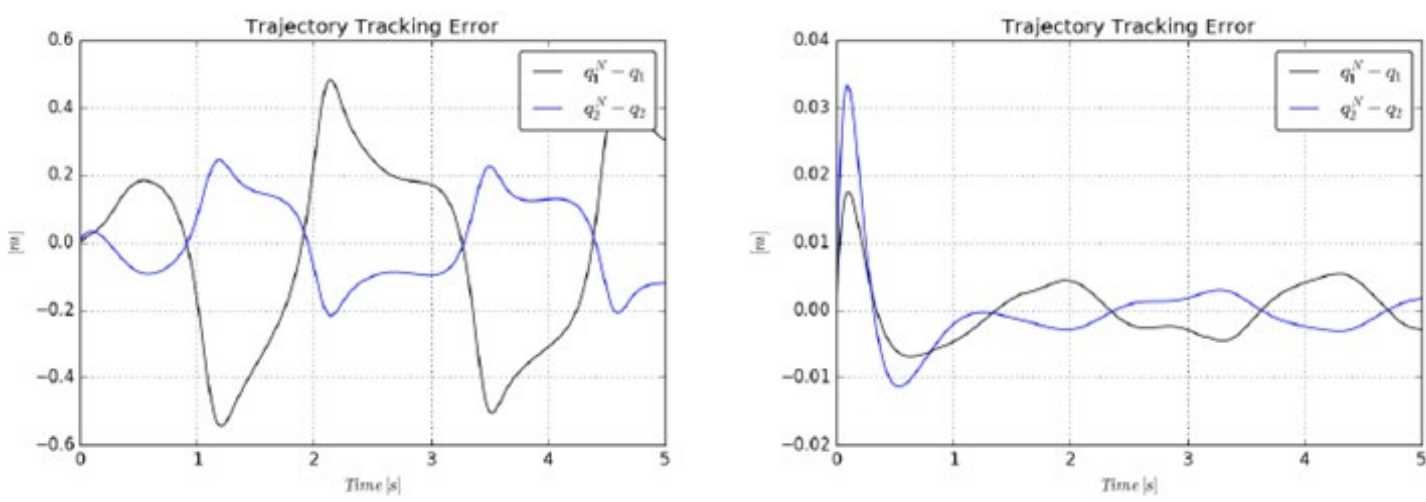

Figure 4: Trajectory tracking error without adaptivity (LHS) and with adaptivity (RHS).
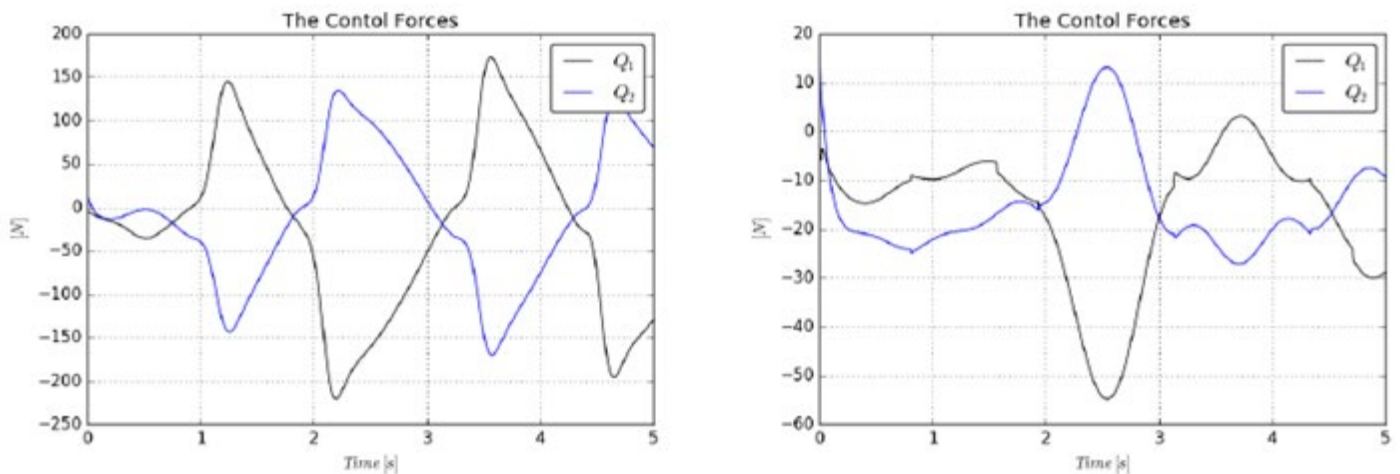

Figure 5: Control force without adaptivity (LHS) and with adaptivity (RHS).
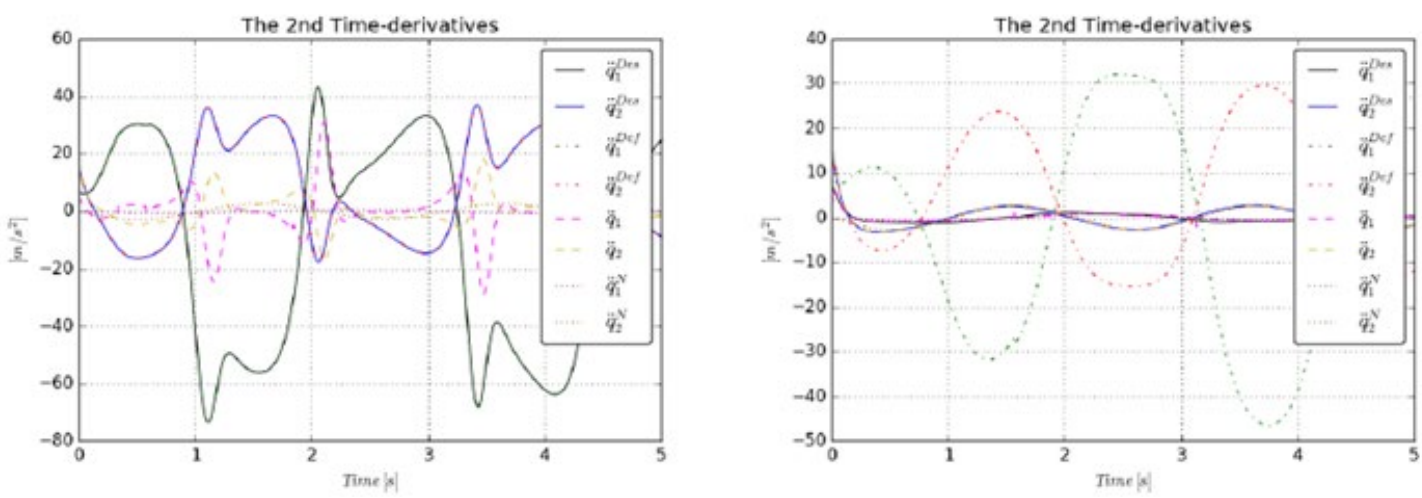

Figure 6: The $2^{\text {nd }}$ time-derivatives without adaptivity (LHS) and with adaptivity (RHS).

The essence of the adaptivity is revealed by Figure 6. In the non-adaptive case considerable PID corrections are added to $\ddot{q}^{N}(t)$ , therefore it considerably differs from $\ddot{q}^{\text {Des }}(t)$ that is identical to $\ddot{q}^{D e s}(t)$ in the lack of adaptive deformation. However, the difference between the desired and the realized $2^{\text {nd }}$ time-derivatives are quite considerable if no adaptive deformation is applied. In contrast to that, in the adaptive case $\ddot{q}^{N}(t)$ is in the vicinity of $\ddot{q}^{\text {Des }}(t)$ because only small PID corrections are needed if the trajectory tracking is precise. This desired value is very close to the realized $2^{\text {nd }}$ time derivatives that considerably differ from the adaptively deformed value. That is, quite considerable adaptive deformation was needed for precise trajectory tracking due to the great modeling errors.

\section{Further Possible Applications and Development}

The applicability of the FPI-based adaptive control design methodology was investigated in various potential fields of application. In 2012 in [55] an adaptive emission control of 
freeway traffic was suggested by the use of the quasistationary solutions of an approximate hydrodynamic traffic model. In [56] an FPI-based adaptive control problem of relative order 4 was investigated. In [57] FPI-based control of the Hodgkin-Huxley Neuron was considered. In [58] the possible regulation of Propofol administration through wavelet-based FPI control in anaesthesia control was investigated.

In [59] the application of the FPI-based control in treating patients suffering from "Type 1 Diabetes Mellitus" was studied. The simplicity of the FPI-based method opened new prospects in the possible design of adaptive optimal controllers. In [60] the contradiction between the various requirements in OC was resolved in the case of underactuated mechanical systems in the following manner: instead constructing a "cost function contribution" to each state variable the motion of which needed control, consecutive time slots were introduced within which only one of the state variables was controlled with FPI-based adaptation. (The different sections may correspond to different relative order control tasks.) In [61] it was pointed out that the FPI-based control can be easily combined with the mathematical framework of the "Receding Horizon Controllers" (RHC) (e.g. [62]). (A combination with the Lyapunov function-based adaptive approach would be far less plausible and simple.) In [49] the applicability of this approach was introduced into the control of systems with time-delay. The possibility of fractional order kinematic trajectory tracking prescription in the FPI-based adaptive control was studied, too [63].

In [64] its applicability was investigated in treating angiogenic tumors. In $[65,66]$ further simplification of the adaptive RHC control was considered in which the reduced gradient algorithm was replace by a FPI

in finding the zero gradient of the "Auxiliary Function" of the problem. In [67] the applicability of the method was experimentally verified in the adaptive control of a pulse-width modulation driven brushless DC motor that did not have satisfactory documentation (FIT0441 Brushless DC Motor with Encoder and Driver) and was braked by external forces simply by periodically grabbing the rotating shaft by one's two fingers. The solution was based on a simple Arduino UNO microcontroller with embedding the adaptive function defined in (3) into the motor's control algorithm. In spite of using $2^{\text {nd }}$ time-derivatives in the feedback no special noise filtering was applied. The measured and computed data was visualized by a common laptop. As it can be seen in Figure 7, the rotational speed was kept at almost constant (in spite of the very noisy measurement data), and the adaptive deformation and the control signal were well adapted to the external braking forces in harmony with the simulation results belonging to the "Illustrative Example" in subsection 2.3.

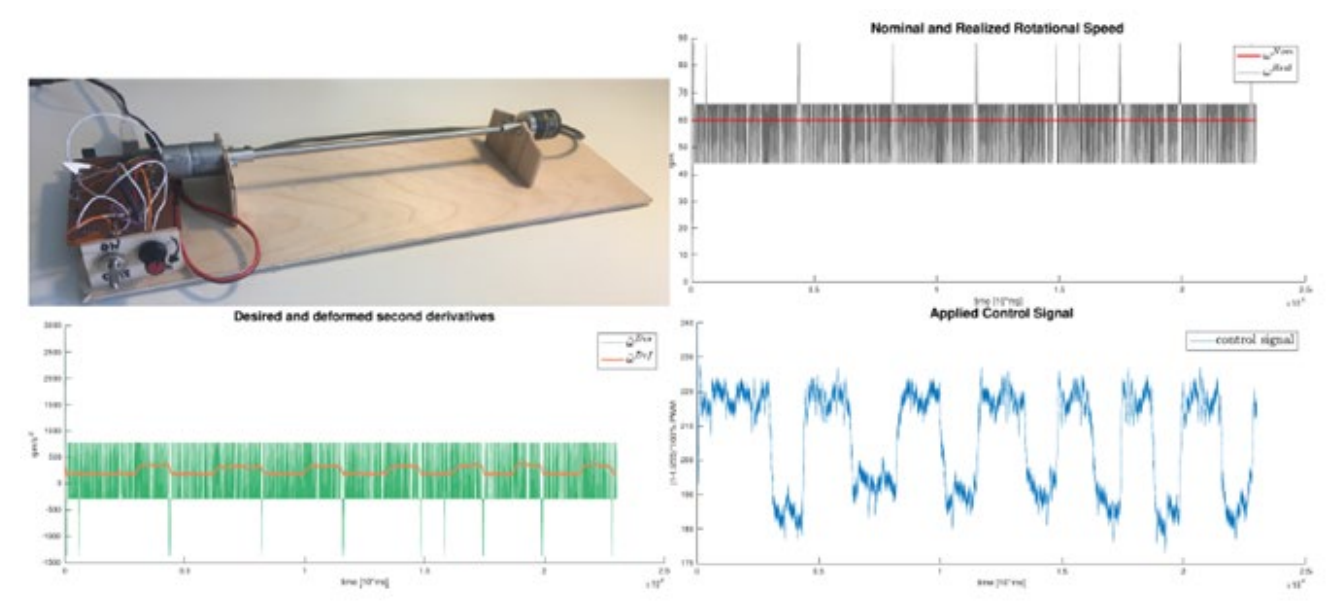

Figure 7: The measured and computed data was visualized by a common laptop.

Figure 7: The experimental setup used for the verification of the FPI-based adaptive control in the case of a pulse-width modulated brushless electric DC motor; The nominal and the realized rotational speed (the average of the whole data set was 59:9383rpm, the nominal constant value was 60rpm); The "Desired" and adaptively "Deformed" $2^{\text {nd }}$ timederivatives of the rotational speed; The control signal (from [67], courtesy of Tamás Faitli) In [68] the novel adaptive control approach was considered from the side of the Lyapunov function-based technique and it was found that it can be interpreted as a novel methodology that is able to drive the Lyapunov function near zero and keeping it in its vicinity afterwards. On this basis a new MRAC controller design was suggested in [69] that has similarity with the idea of the "Backstepping Controller" [70,71].

\section{Conclusion}

The FPI-based adaptive control approach was introduced at the Óbuda University with the aim of evading the mathematical difficulties and restrictions, furthermore the information need related to the traditional Lyapunov function-based design. Its 
main point was the transformation of the control task into a fixed-point problem that was iteratively solved on the firm mathematical basis of Banach's fixed point theorem. In the center of the new approach, instead of the requirement of global stability, as the primary design intent, precise realization of a kinematically (kinetically) prescribed tracking error relaxation was placed. In contrast to the traditional soft computing approaches as fuzzy, neural network and neuro-fuzzy solutions that normally apply huge structures with ample number of the parameters of the universal approximators of the continuous multiple variable functions on the basis of Kolmogorov's approximation theorem (e.g.

[72-74]) this approach has only a few independent adaptive parameters that can be easily set and one of them can be tuned for maintaining the convergence of the control algorithm. It was shown that the simplicity of this approach allows its combination with more "traditional" approaches as that learning the exact model parameters of the controlled system and at various levels of the optimal controllers as the RFC control. On the basis of ample simulation investigations, it can be stated that the suggested approach has wide area of potential applications (in the control of mechanical devices, in life sciences, traffic control, etc.) where the presence of essential nonlinearities, the lack of precise and complete system models, and limited possibilities for obtaining information on the controlled system's state are present as main difficulties. It seems to be expedient to invest more efforts into experimental investigations.

\section{Acknowledgement}

The Authors express their gratitudes to the Antal Bejczy Center for Intelligent Robotics, and the Doctoral School of Applied Informatics and Applied Mathematics for supporting their work.

\section{References}

1. Armstrong B, Khatib O, Burdick J (1986) The explicit dynamic model and internal parameters of the PUMA 560 arm. Proc IEEE Conf on Robotics and Automation pp. 510-518.

2. Corke PI, Armstrong Helouvry B (1994) A search for consensus among model parameters reported for the PUMA 560 robot. Proc IEEE Conf Robotics and Automation pp. 1608-1613.

3. Pacejka HB, Bakker E (1993) The magic formula tyre model. Vehicle System Dynamics 21: 1-18.

4. van Oosten JJM, Bakker E (1993) Determination of magic tyre model parameters. Vehicle System Dynamics 21: 19-29.

5. Lidner L (1993) Experience with the magic formula tyre model. Vehicle System Dynamics 21: 30-46.

6. Svendenius J (2003) The tire models for use in braking applications. Department of Automatic Control, Lund Institute of Technology, Sweden.

7. Hirschberg W, Rill G, Weinfurter H (2007) Tire model tmeasy. Vehicle System Dynamics 45: 101-119.

8. Bergman RN, Ider YZ, Bowden CR, Cobelli C (1979) Quantitative estimation of insulin sensitivity. Am J Physiol 236: E667-E677.
9. Ni TC, Ader M, Bergman EN (1997) Reassessment of glucose effectiveness and insulin sensitivity from minimal model analysis: a theoretical evaluation of the single-compartment glucose distribution assumption. Diabetes 46: 1813-1821.

10. Hovorka R, Shojaee Moradie F, Carroll PV, Chassin LJ, Gowrie IJ, et al (2002) Partitioning glucose distribution/transport, disposal, and endogenous production during ivgtt. Am J Physiol Endocrinol Metab 282: E992-E1007.

11. Friis Jensen E (2007) Modeling and Simulation of Glucose-Insulin Metabolism. Technical University of Denmark, Kongens Lyngby, Denmark.

12. Ionescu CM, De Keyser R, Torrico CB, Smet TD, Struys MMRF, et al. (2008) Robust predictive control strategy applied for Propofol dosing using BIS as a controlled variable during anesthesia. IEEE Transactions on Biomedical Engineering 55(9): 2161-2170.

13. Na I, scu A, Krieger CM, Ionescu, Pistikopoulos EN (2014) Advanced model-based control studies for the induction and maintenance of intravenous anaesthesia. IEEE Transactions on Biomedical Engineering 62(3): 832-841.

14. Ionescu CM, Na I scu De, Keyser R (2014) Lessons learned from closed loops in engineering: towards a multivariable approach regulating depth of anaesthesia. J Clin Monit Comput 28(6): 537-546.

15. Foz o L, Andoga R, Madarász L (2010) Mathematical model of a small Turbojet Engine MPM-20. Computational Intelligence in Engineering 313: 313-322.

16. Andoga R, Foz o L, Madarász L, Karol T (2013) A digital diagnostic system for a small turbojet engine. Acta Polytechnica Hungarica, 10(4): 45-58.

17. Andoga R, Madarász L, Foz o L, Lazar T, Gašpar V (2013) Innovative approaches in modeling, control and diagnostics of small turbojet engines. Acta Polytechnica Hungarica 10(5): 81-99.

18. Ladislav Nyulászi, Rudolf Andoga, Peter Butka, Ladislav Foz o, Radovan Kovacs, et al. (2018) Fault detection and isolation of an aircraft turbojet engine using a multi-sensor network and multiple model approach. Acta Polytechnica Hungarica 15(2): 189-209.

19. Jean Jacques E Slotine, Li W (1991) Applied Nonlinear Control. Prentice Hall International Inc, Englewood Cliffs, New Jersey, USA.

20. Nguyen CC, Antrazi SS, Zhen Lei Zhou, Campbell CE (1993) Adaptive control of a Stewart platform-based manipulator. Journal of Robotic Systems 10(5): 657-687.

21. Somló J, Lantos B, Cát PT (2002) Advanced Robot Control. Akadémiai Kiadó, Budapest.

22. Hosseini Suny K, Momeni H, Janabi-Sharifi F (2010) Model Reference Adaptive Control design for a teleoperation system with output prediction. J Intell Robot Syst 59(2): 319-339.

23. Lyapunov AM (1892) A General Task about the Stability of Motion (in Russian). Thesis, University of Kazan, Tatarstan, Russia.

24. Lyapunov AM (1966) Stability of Motion. Academic Press, New-York and London.

25. Tar JK, Bitó JF, Nádai L, Tenreiro Machado JA (2009) Robust Fixed point Transformations in adaptive control using local basin of attraction. Acta Polytechnica Hungarica 6(1): 21-37.

26. Tjalling J Ypma (1995) Historical development of the Newton-Raphson method. SIAM Review 37(4): 531-551.

27. Kelley CT (2003) Solving Nonlinear Equations with Newton's Method, no 1 in Fundamentals of Algorithms. SIAM. 


\section{Robotics \& Automation Engineering Journal}

28. Deuflhard P (2004) Newton Methods for Nonlinear Problems. Affine Invariance and Adaptive Algorithms, Springer Series in Computational Mathematics 35 .

29. Banach S (1922) Sur les opérations dans les ensembles abstraits et leur application aux équations intégrales (About the Operations in the Abstract Sets and Their Application to Integral Equations) Fund Math 3(1): 133-181.

30. Hazewinkel M (Ed)(1994) Encyclopaedia of Mathematics (set). Kluwer

31. Campa R, Kelly R, García E (2001) On stability of the resolved acceleration control. In Proc of the 2001 IEEE International Conference on Robotics \& Automation pp. 3523-3528.

32. Musa Mailah, Endra Pitowarno, Hishamuddin Jamaluddin (2005) Robust motion control for mobile manipulator using resolved acceleration and proportional-integral active force control. International Journal of Advanced Robotic Systems 2(2): 125-134.

33. Behzad Dariush, Ghassan Bin Hammam, David Orin (2018) Constrained resolved acceleration control for humanoids. In Proc of the 2010 IEEE/ RSJ International Conference on Intelligent Robots and Systems, pp 710-717.

34. Karl Petter W Lindegaard (2003) Acceleration Feedback in Dynamic Positioning (PhD Dissertation). Department of Engineering Cybernetics, Norwegian University of Science and Technology, Trondheim, Norway.

35. Dumetz E, Jean Yves Dieulot, Pierre Jean Barre, Colas F, Delplace T (2006) Control of an industrial robot using acceleration feedback. Journal of Intelligent and Robotic Systems 46(2): 111-128.

36. Xu Wl, Han JD (2000) Joint acceleration feedback control for robots: analysis, sensing and experiments. Robotics and Computer Integrated Manufacturing 16: 307-320.

37. Xu WL, Han JD, Tso SK (2000) Experimental study of contact transition control incorporating joint acceleration feedback. IEEE/ASME Transactions on Mechatronics 5(3): 292-301.

38. Wang Q Hua Xiang C, Mei Huang Y, Liang Ge, Tang T, et al. (2016) Acceleration feedback control (AFC) enhanced by disturbance observation and compensation (DOC) for high precision tracking in telescope systems. Research in Astronomy and Astrophysics 16(8): 124.

39. Tar JK, Nádai L, Rudas IJ, Várkonyi TA (2011) RFPT based adaptive control stabilized by fuzzy parameter tuning. In Proc of the $9^{\text {th }}$ European Workshop on Advanced Control and Diagnosis (ACD 2011), Budapest, Hungary, p. 1-8.

40. Kósi K, Hajdu Sz, Bitó JF, Tar JK (2012) Chaos formation and reduction in Robust Fixed-Point Transformations based adaptive control. In Proc. of the 4th IEEE Intl. Conf. on Nonlinear Science and Complexity (NSC 2012), Budapest, Hungary, pp. 211-216.

41. Kósi K, Breier A, Tar JK (2012) Chaos patterns in a 3 degree of freedom control with robust fixed-point transformation. In Proc of the $13^{\text {th }}$ IEEE Intl. Symp on Computational Intelligence and Informatics, Budapest, Hungary, pp. 1-5.

42. Tar JK, Bitó JF, Rudas IJ (2010) Replacement of Lyapunov's Direct Method in Model Reference Adaptive Control with Robust FixedPoint Transformations. In Proc of the $14^{\text {th }}$ IEEE Intl Conf on Intelligent Engineering Systems, Las Palmas of Gran Canaria, Spain, pp 231-235.

43. Tar JK, Bitó JF, Rudas IJ, Eredics K (2010) Comparative analysis of a traditional and a novel approach to Model Reference Adaptive Control. $11^{\text {th }}$ Intl Symp of Hungarian Researchers on Computational Intelligence and Infor matics, Budapest, Hungary,pp 93-98.

44. Tar JK, Rudas IJ, BitóJF, Kósi K (2013) Robust fixed-pointtransformations in the model reference adaptive control of a three DoF aeroelastic wing Applied Mechanics and Materials 300-301: 1505-1512.
45. Tar JK, Kovács L, Takács A, Takács B, Zentay P, et al. (2014) Novel design of a Model Reference Adaptive Controller for soft tissue operations. 2014 IEEE International Conference on Systems, Man, and Cybernetics San Diego, CA, USA, pp. 2476-2481.

46. Tar Jk, Várkonyi TA, Kovács L, Rudas IJ, Haidegger T (2014) Robust Fixed-Point Transformation based design for Model Reference Adaptive Control of a modified TORA system Proc of the 2014 IEEE/ RSJ International Conference on Intelligent Robots and Systems (IROS 2014), Chicago, USA, pp: 123-128.

47. Tar JK, Rudas IJ, Dineva A, Várkonyi-Kóczy A (2014) Stabilization of a Modified Slotine-Li Adaptive Robot Controller by Robust Fixed-Point Transformations. In Proc. of Recent Advances in Intelligent Control, Modelling and Simulation Cambridge, MA, USA, pp. 35-40.

48. Dineva A, Várkonyi-Kóczy AR, Tar JK (2014) Combination of RFPT based adaptive control and classical model identification. In Proc. of the IEEE $12^{\text {th }}$ Intl Symp on Applied Machine Intelligence and Informatics (SAMI 2014), 2014, Herl'any, Slovakia, pp 35-40.

49. Redjimi H, Tar JK (2017) On the effects of time-delay on precision degradation in fixed point transformation-based adaptive control. In the Proc of the 2017 IEEE 30th Jubilee Neumann Colloquium, Budapest, Hungary, pp. 125-130.

50. Dineva A, Tar JK, Várkonyi Kóczy AR (2015) Novel generation of FixedPoint Transformation for the adaptive con-trol of a nonlinear neuron model. In proc. of the IEEE International Conference on Systems, Man, and Cybernetics, Hong Kong, pp 987-992.

51. Dineva A, Tar JK, Várkonyi-Kóczy AR, Piuri V (2015) Generalization of a Sigmoid Generated Fixed-Point Trans-formation from SISO to MIMO systems. In Proc. of the IEEE $19^{\text {th }}$ International Conference on Intelligent Engineering Systems pp. 35-140.

52. Dineva A (2016) Non-conventional Data Representation and Control. Óbuda University, Budapest, Hungary.

53. Utkin VI (1992) Sliding Modes in Optimization and Control Problems. Springer Verlag, New York, USA.

54. Levant A (1998) Arbitrary-order sliding modes with finite time convergence. In Proc. of the 6th IEEE Mediterranean Conference on Control and Systems, Alghero, Sardinia, Italy.

55. JK Tar, Nádai L, Rudas IJ, Várkonyi TA (2012) Adaptive emission control of freeway traffic using quasi-stationary solutions of an approximate hydrodynamic model. Journal of Applied Nonlinear Dynamics 1(1): 2950 .

56. Kósi K, Várkonyi TA, Tar JK, Rudas IJ, Bitó JF (2013) On the simulation of RFPT-based adaptive control of systems of 4th order response. In Proc of the IEEE $11^{\text {th }}$ Intl Symp on Intelligent Systems and Informatics (SISY 2013) Subotica, Serbia, pp. 259-264.

57. Bitó JF, Tar JK (2015) Fixed point transformation-based adaptive control of the Hodgkin-Huxley neuron. Proc of the Workshop on Information Technology and Bionics - Symposium in Memory of Tamás Roska, Pázmány University ePress, Budapest, Hungary, pp. 69-72.

58. Dineva A, Tar JK, Vákonyi-Kóczi AR, Piuri V (2016) Adaptive controller using Fixed Point Transformation for regulating Propofol administration through wavelet-based anesthetic value. In Proc of the IEEE International Symposium on Medical Measurements and Applications (MeMeA 2016) Benevento, Italy, pp. 650-655.

59. Eigner Gy, Horváth P, Tar JK, Rudas IJ, Kovács L (2015) Application of Robust Fixed Point control in case of T1DM. In proc. of the IEEE International Conference on Systems, Man, and Cybernetics, Hong Kong (SMC 2015) pp. 2459-2463.

60. Tar JK, Bitó JF, Rudas IJ (2016) Contradiction resolution in the adaptive control of underactuated mechanical systems evading the framework of optimal controllers. Acta Polytechnica Hungarica 13(1): 97-121. 
61. Khan H, Szeghegyi A, Tar JK (2017) Fixed point transformation-based adaptive optimal control using NP. In Proc. of the 2017 IEEE 30th Jubilee Neumann Colloquium Budapest, Hungary, pp. 35-40.

62. Richalet A, Rault JL, Testud, Papon J (1978) Model predictive heuristic control: Applications to industrial processes. Automatica 14(5): 413428

63. Tar JK, Bitó JF, Kovács L, Faitli T (2018) Fractional order PID-type feedback in Fixed Point Transformation-based adaptive control of the FitzHugh-Nagumo neuron model with time-delay. In 3rd IFAC Conference on Advances in Proportional-Integral-Derivative Control Ghent, Belgium, pp. 906-911.

64. Kovács L, Eigner Gy, Tar Jk, Rudas I (2018) Robust fixed-point transformation based proportional-derivative control of angiogenic tumor growth. In $3^{\text {rd }}$ IFAC Conference on Advances in ProportionalIntegral-Derivative Control, Ghent, Belgium, pp. 894-899.

65. Khan H, Tar JK, Rudas IJ, Eigner Gy (2017) Adaptive model predictive control based on fixed point iteration. WSEAS Transactions on Systems and Control 12: 347-354.

66. Khan H, Tar JK, Rudas IJ, Eigner Gy (2018) Iterative solution in Adaptive Model Predictive Control by using Fixed-Point Transformation method. International Journal of Mathematical Models and Methods in Applied Sciences 12: 7-15.

67. Faitli T (2018) Investigation of Control Methods for a Speed-controlled Electric Motor. Óbuda University, Donát Bánki Faculty of Mechanical and Safety Engineering, Institute of Mechatronics and Autotechnics, Budapest, Hungary.

68. Csanádi B, Galambos P, Tar JK, Györök Gy, Serester A (2018) Revisiting Lyapunov's technique in the Fixed-Point Transformationbased adaptive control. Accepted for publication at the 22nd IEEE International Conference on Intelligent Engineering Systems, Las Palmas de Gran Canaria, Spain, pp. 1-6.

69. Csanádi B, Tar JK Novel Model Reference Adaptive Control designed by a Lyapunov function that is kept at low value by Fixed Point Iteration. Submitted for publication to Springer.

70. Krstic M, Kanellakopoulos I, Kokotovic PV (1995) Nonlinear and Adaptive Control Design. Wiley Interscience, New York, USA.

71. Khalil HK (1996) Nonlinear Systems (2 ${ }^{\text {nd }}$ edn), Prentice Hall, Upper Saddle River, USA.

72. Kolmogorov AN (1957) On the representation of continuous functions of many variables by superposition of continuous functions of one variable and addition (in russian) Dokl Akad Nauk SSSR 114: 953-956.

73. Sprecher DA (1965) On the structure of continuous functions of several variables. Trans Amer Math Soc 115: 340-355.

74. Lorentz GG (1976) Mathematical Developments Arising from Hilbert's Problems 2. American Mathematical Society, Providence pp. 419-430.

\section{Your next submission with Juniper Publishers} will reach you the below assets

- Quality Editorial service

- Swift Peer Review

- Reprints availability

- E-prints Service

- Manuscript Podcast for convenient understanding

- Global attainment for your research

- Manuscript accessibility in different formats

( Pdf, E-pub, Full Text, Audio)

- Unceasing customer service

Track the below URL for one-step submission https://juniperpublishers.com/online-submission.php 